\title{
Thresholds Optimization of Decomposed Vector Rotation Model for Digital Predistortion of RF Power Amplifier
}

\author{
Chouaib KANTANA, Geneviève BAUDOIN, Olivier VENARD \\ ESYCOM, Univ Gustave Eiffel, CNRS, CNAM, ESIEE Paris, F-77454 Marne-la-Vallee, France \\ chouaib.kantana@esiee.fr
}

Submitted October 1, 2020 / Accepted January 3, 202

\begin{abstract}
In this paper, we propose an efficient approach for optimizing the decomposed vector rotation (DVR) model for digital predistortion (DPD). The DVR model's basis functions are constructed piecewise by dividing the input space into segments bounded by thresholds. This paper investigates how to set the thresholds optimally using an iterative approach based on the decomposition of the global optimization problem into a set of unimodal sub-problems so that a unidirectional minimization can be used to optimize the positions of thresholds. The proposed approach has been evaluated using measurements from a real power amplifier (PA). The experimental results illustrate the efficiency of the proposed optimization approach and show that the thresholds' optimization improves linearization performances significantly compared to conventional DVR with uniform segmentation.
\end{abstract}

\section{Keywords}

Power amplifiers, digital predistortion, DVR, optimization of thresholds.

\section{Introduction}

Higher data rates require to use spectral efficient modulation techniques such as Orthogonal Frequency Division Multiplexing (OFDM). As a result, the modulated signals have a high peak-to-average power ratio (PAPR), which stimulates PA's nonlinearities. These nonlinearities introduce inband and out-of-band distortions that cause spectral regrowth causing interference between channels.

Digital predistortion is a powerful technique used to linearize PA and compensate for these distortions. This operation allows to pre-compensate the nonlinear effects of the PA, and the behavior of the whole system becomes linear and memoryless [1,2].

Several mathematical models have been proposed for DPD. Most of them are derived from Volterra Series model [3-5]. However, other approaches, such as the segmentation approach, consist of dividing the amplitude range into segments and applying a specific nonlinear function to each of them. The segmentation approach can represent strong non-nonlinearities and may have good numerical properties regarding their implementation [6].

In [7], the author proposed a behavioral model based on Decomposed Vector Rotation (DVR). The DVR model structure is determined by the number of segments $K$ bounded by thresholds $\beta_{k}$ and by the set of basis functions that are applied to each segment.

The thresholds $\beta_{k}$ that define the boundaries of segments can be selected using uniform or optimized segmentation. Although many works in the literature are dedicated to the DVR model with uniform segmentation, few of them deal with optimal segmentation.

The first work for DPD application about optimal spacing was conducted for memoryless LUT DPD [8]. The author has proposed a non-uniform spacing using a companding function into table indexing. The benefit of optimal segmentation versus uniform segmentation has already been established in [9] where the authors suggest to reduce the complexity of the algorithm by considering the memoryless version of the actual DVR model. Nevertheless, even with the reduced complexity algorithm proposed in [9], the problem to solve is still a nonlinear least square problem. The authors claim that the nonlinear minimization problem behaves well close to uniform segmentation and can be handled with Gauss-Newton based methods.

Generally speaking, setting the thresholds optimally for piecewise models such as the DVR model requires global optimization, and in [10] an approach based on genetic algorithm (GA) has been used to optimize the thresholds of a canonical piecewise linear (CPWL) model.

In this paper, we propose a new approach in which the optimization problem is decomposed into a set of unimodal sub-problems that allow to use unidirectional minimization and decrease the search complexity dramatically. The proposed approach has been compared to GA and brute-force in terms of linearization performance and search complexity to validate its effectiveness.

In the following, we present the structure of the DVR 
model in Sec. 2. In Sec. 3, the test bench is presented. The proposed approach to optimize the thresholds of the DVR model is described in Sec. 4. The results are discussed and analyzed in Sec. 5. Finally, Section 6 gives a conclusion.

\section{DVR Model}

The DVR model is an extension of the CPWL model, which is capable of representing a wide range of continuous nonlinear functions with high precision [11].

The basis functions of the DVR model are constructed piecewise using the decomposed vector rotation technique, which allows the model to handle complex signals and nonlinear systems with memory. Besides, the DVR model is linear with respect to its coefficients. The DVR model can include different terms [7]. For example using " $1^{\text {st }}$ and " $2^{\text {nd }}$ " order terms, the DVR model can be defined as:

$$
\begin{aligned}
y(n) & =\sum_{i=0}^{M} a_{i} x(n-i) \\
& +\sum_{k=1}^{K} \sum_{i=0}^{M} c_{k i, 1}|| x(n-i)\left|-\beta_{k}\right| \mathrm{e}^{\mathrm{j} \theta(n-i)} \\
& +\sum_{k=1}^{K} \sum_{i=0}^{M} c_{k i, 2}|| x(n-i)\left|-\beta_{k}\right| \mathrm{e}^{\mathrm{j} \theta(n-i)}|x(n)| \\
& +\sum_{k=1}^{K} \sum_{i=1}^{M} c_{k i, 3}|| x(n-i)\left|-\beta_{k}\right| x(n) \\
& +\sum_{k=1}^{K} \sum_{i=1}^{M} c_{k i, 4}|| x(n-i)\left|-\beta_{k}\right| x(n-i) \\
& +\sum_{k=1}^{K} \sum_{i=1}^{M} c_{k i, 5}|| x(n)\left|-\beta_{k}\right| x(n-i)
\end{aligned}
$$

where $x(n)$ and $y(n)$ are the baseband input and output signals, respectively. $M$ represents the memory depth. $a_{i}$ and $c_{k i, n}$ are the model coefficients. $K$ is the number of segments, and $\beta_{k}$ are the thresholds.

The coefficients of the DVR model depend on the memory depth and the number of segments. According to (1), the number of coefficients $C$ is defined by:

$$
C=(1+M)(1+2 K)+3 K M .
$$

The coefficients $C$ are identified using postdistorter from the indirect learning architecture (ILA), as shown in Fig. 1. The ILA aims is to minimize the difference between the output of the postdistorter $z_{\mathrm{p}}$ and the input of the PA $x$. The error is thus defined as $e(n)=x(n)-z_{\mathrm{p}}(n)$.

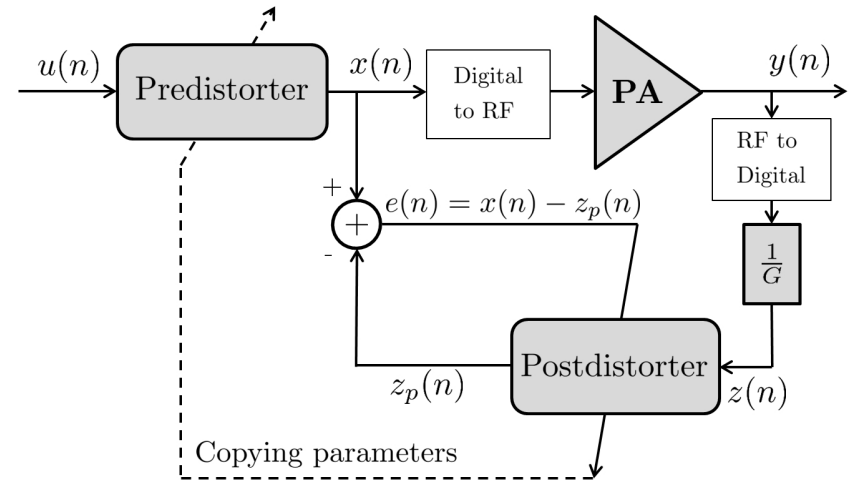

Fig. 1. Indirect learning architecture.

The identification of the model coefficients using a leastsquare criterion is obtained by solving the normal equations

$$
\left[\mathbf{Z}^{\mathrm{H}} \mathbf{Z}\right] \mathbf{c}=\mathbf{Z}^{\mathrm{H}} \mathbf{x}
$$

where $(.)^{\mathrm{H}}$ represents the Hermitian transpose, $x$ is the input vector, $\mathbf{Z}$ is the matrix of regressors, and $\mathbf{c}$ is the vector of coefficients.

To evaluate the modeling accuracy of the DVR model, the NMSE is calculated between the post-distortion output $z_{\mathrm{p}}(n)$ and the PA input signal $x(n)$.

$$
\mathrm{NMSE}_{\mathrm{dB}}=10 \log _{10} \frac{\sum_{n=1}^{N}\left|x(n)-z_{\mathrm{p}}(n)\right|^{2}}{\sum_{n=1}^{N}|x(n)|^{2}} .
$$

From a system point of view, the figure of merit is provided by the out-band distortions, which are evaluated using adjacent channel power ratio (ACPR), which are defined for the right and left channel as

$$
\begin{aligned}
& \mathrm{ACPR}_{\mathrm{R}, \mathrm{dB}}=10 \log _{10} \frac{\int_{-B / 2}^{B / 2} P(y(t)) \mathrm{dt}}{\int_{B / 2}^{3 B / 2} P(y(t)) \mathrm{dt}}, \\
& \mathrm{ACPR}_{\mathrm{L}, \mathrm{dB}}=10 \log _{10} \frac{\int_{-B / 2}^{B / 2} P(y(t)) \mathrm{dt}}{\int_{-3 B / 2}^{-B / 2} P(y(t)) \mathrm{dt}},
\end{aligned}
$$

where $B$ represents the bandwidth of the signal, and $P($.$) is$ power spectral density.

\section{Experimental Test Bench}

\subsection{Test Bench}

Experiments are carried out using measurements from the test bench represented in Fig. 2. 


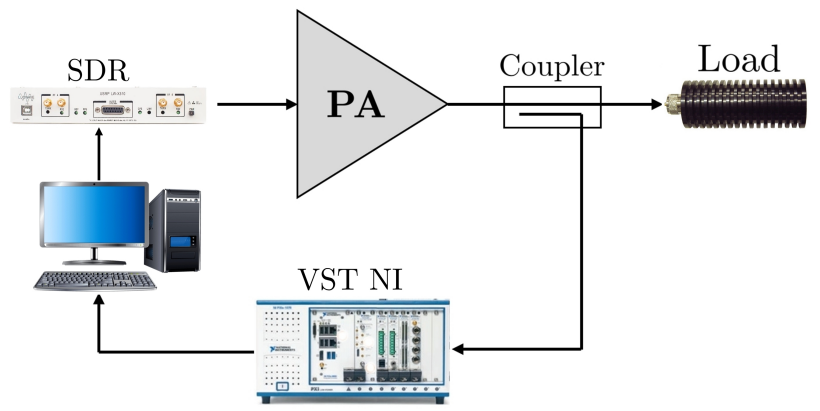

Fig. 2. Test bench block diagram.

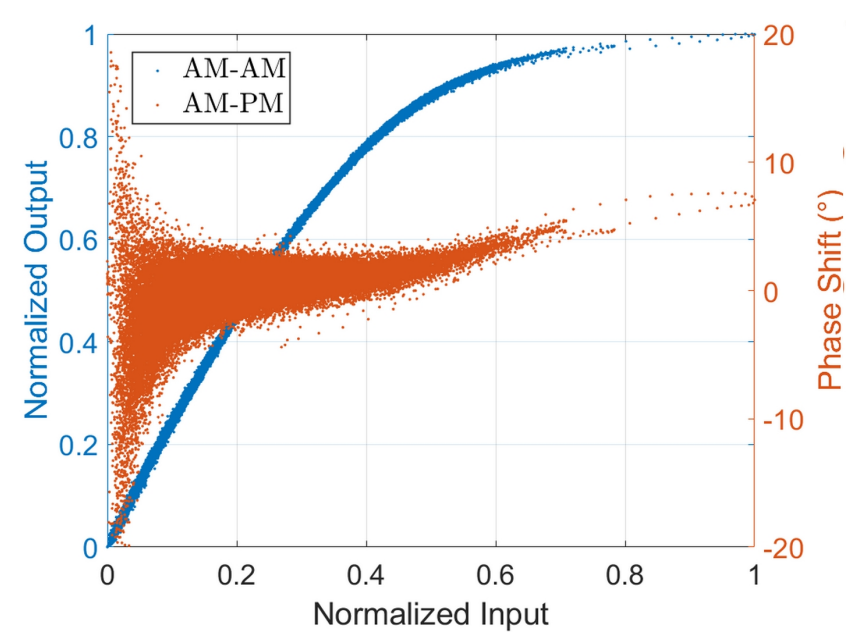

Fig. 3. AM-AM and AM-PM curves of PA.

The baseband IQ signal is fed from a software-defined radio (SDR) board manufactured by ARELIS to an LDMOS $\mathrm{PA}$ at a carrier frequency of $1.8 \mathrm{GHz}$. The output signal is captured through a coupler and then down-converted and acquired by a vector signal transceiver (VST). The PA is excited by a $10 \mathrm{MHz}$ LTE signal with an effective PAPR of $9 \mathrm{~dB}$. The average output power of the PA is $44 \mathrm{dBm}$. Around 64000 IQ samples are used for the identification process. Adjacent channel power ratio (ACPR) and normalized mean square error (NMSE) were used for performance evaluation.

The non-nonlinearities of the PA can be seen from the AM-AM and AM-PM curves for the LTE signal in Fig. 3.

According to Figure 3, the AM-AM characteristic's behavior is not typical over the interval [0.8 1], where there are very few data points located in the high power region.

Indeed, a crest factor reduction (CFR) could be applied to clip the input signal by setting the clipping threshold to 0.8 , but as we are concerned in this study by the optimization of the thresholds which strongly depends on the input range, we note that no CFR has been applied.

\subsection{Baseband Postdistortion}

In this study, the effectiveness of the proposed approach to optimize the DVR model thresholds has been validated using the postdistortion architecture from baseband using Matlab software.

In this process, the coefficients $C$ are estimated, but without copying them to the predistorter model, which means that only the input-output signals from PA are required. For real-time DPD adaptation, the proposed approach is only required to be performed at the initial training when the global characteristics of the PA are determined to selected the DPD model.

Therefore, the proposed approach is seen as an offline process to tune the DVR model by optimizing its thresholds $\beta$.

\section{Optimization of DVR Model}

\subsection{Motivation}

The benefits of an optimized segmentation is highlighted in Fig. 4, which compares uniform and optimal segmentation for different number of segments in terms of NMSE for a $10 \mathrm{MHz}$ bandwidth LTE signal with a given DVR model. According to Fig. 4, the linearization performance for 8 segments using uniform segmentation could be achieved with only 3 segments using optimal segmentation.

The NMSE of the DVR model with optimal segmentation converges towards an asymptote from 4 segments, while uniform segmentation requires more than 10 segments to reach the best linearization performance.

Thus, the optimal segmentation significantly reduces the complexity since the number of coefficients to be estimated is reduced according to (2). This confirms the interest in optimizing the DVR model thresholds.

In the sequel, we present the proposed approach to optimize the thresholds $\beta_{K}$ and how the global optimization problem could be divided into a set of unimodal sub-problems requiring only a unidirectional minimization.

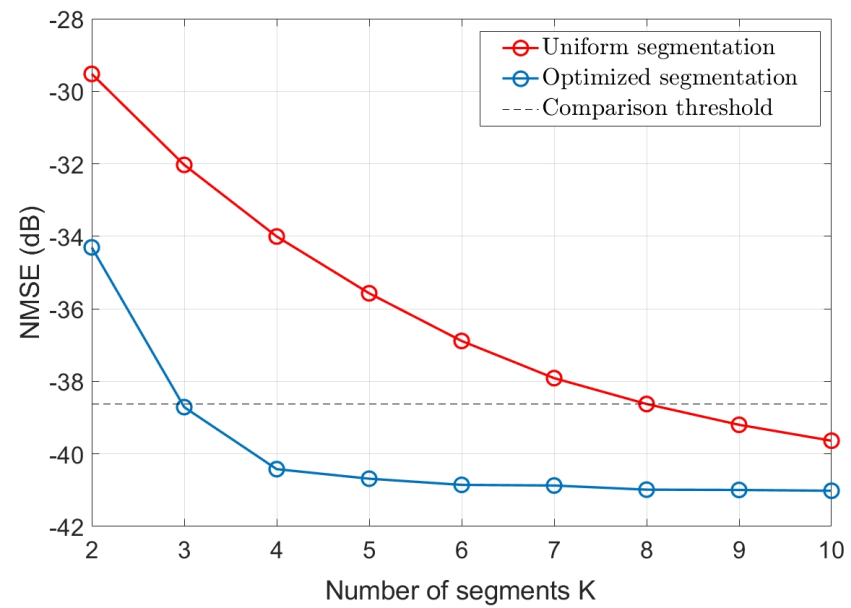

Fig. 4. NMSE versus number of segments $K$ with $M=2$. 


\subsection{Principle of Unidirectional Minimization}

In the proposed approach, unidirectional minimization is achieved using the golden section (GS) algorithm.

The GS search is an optimization technique used to find the optimum for a strictly unimodal function of a single variable over a search interval without using derivatives [12]. For a given unimodal function $f(x)$, the principle of GS search consists of finding the optimum $x_{\mathrm{opt}}$ which corresponds to the minimum value of $f(x)$, by iteratively narrowing the range of the search interval $\left[\begin{array}{ll}a & b\end{array}\right]$ containing that optimum until a specified accuracy is reached. The GS search derives its name from the fact that the points determining the search interval are computed using the golden ratio $\tau$, which is equal to $\frac{1+\sqrt{5}}{2} \approx 1.618$ and verifies $\tau-1=\frac{1}{\tau}$. In fact, the golden ratio $\tau$ is intimately involved in the Fibonacci sequence, in which the ratios of successive terms of the Fibonacci sequence possess an impressive proportion, e.g., 1.618, or its inverse 0.618 .

Therefore, the length of the search interval is iteratively reduced by a factor $\tau$. At q-th iteration the search interval is noted $\left[a_{q} b_{q}\right]$. Denoting $\varphi=\frac{1}{\tau}$, two intermediate points are used to update the range of the search interval. They are determined by

$$
\left\{\begin{array}{l}
x_{1}=a_{q}+\varphi^{2}\left(b_{q}-a_{q}\right) \\
x_{2}=a_{q}+\varphi\left(b_{q}-a_{q}\right)
\end{array} .\right.
$$

The function value $f\left(x_{1}\right)$ and $f\left(x_{2}\right)$ are computed and compared as described in Algorithm (1).

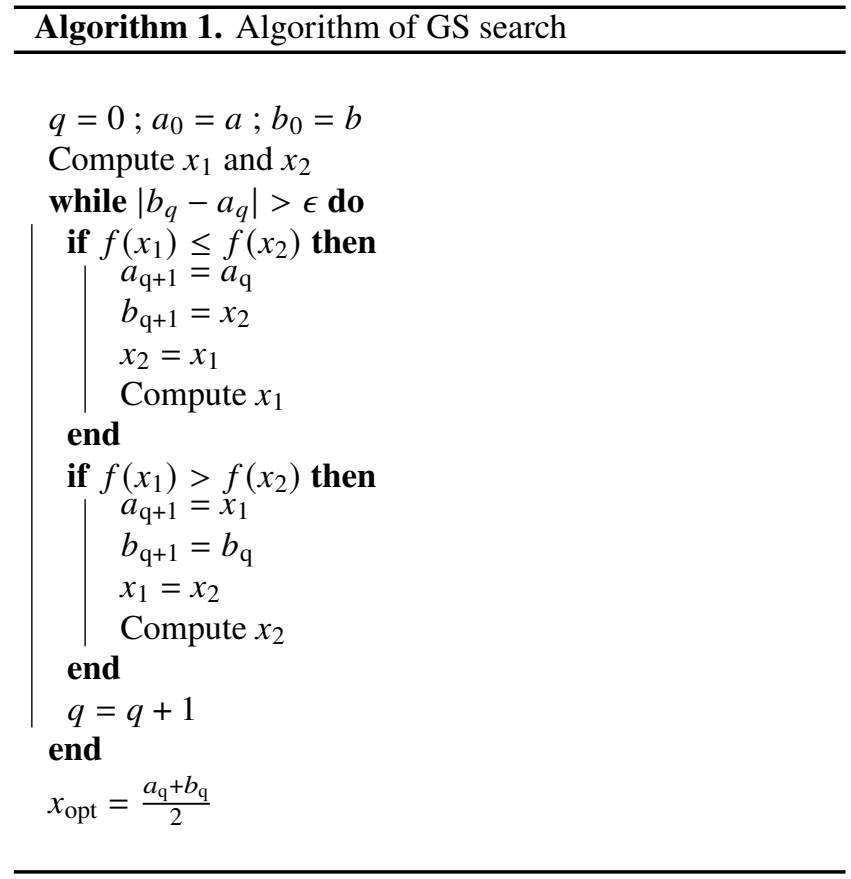

The computation process of $f\left(x_{1}\right)$ and $f\left(x_{2}\right)$ is iteratively executed and the search interval $\left[\begin{array}{ll}a & b\end{array}\right]$ is narrowing until the accuracy $\left|b_{\mathrm{q}}-a_{\mathrm{q}}\right|<\epsilon$ is reached where $\epsilon$ is a specified small value, then takes $x_{\mathrm{opt}}=\frac{a_{\mathrm{q}}+b_{\mathrm{q}}}{2}$ as an approximate optimum point and $f\left(x_{\mathrm{opt}}\right)$ is an approximate optimum value.

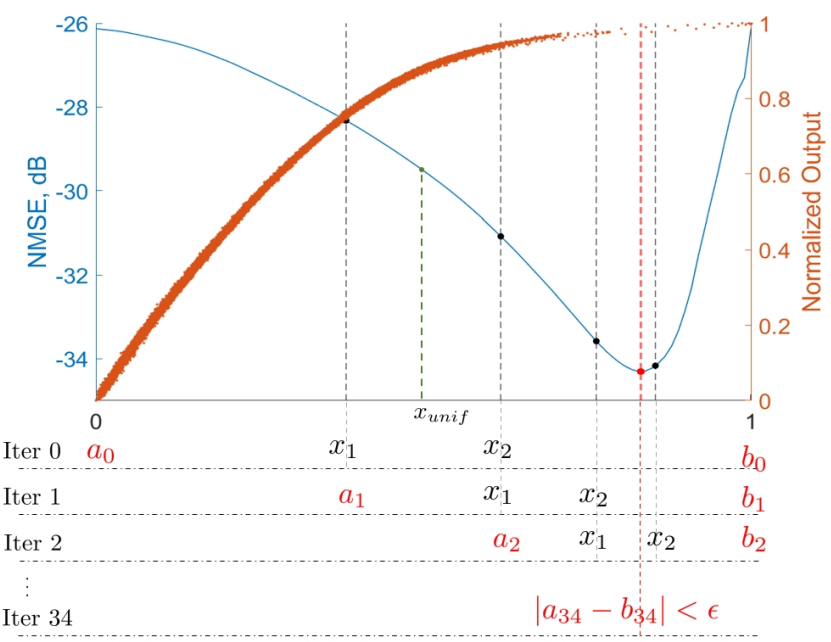

Fig. 5. Evolution of GS search with $\epsilon=0.1$

In this work, the GS search NMSE as the function $f(x)$ and the range of the normalized magnitude of the baseband input IQ as the search interval $[a b]$.

Another advantage of the GS search is that it requires only one computation of $f(x)$ at each iteration, except for the first one where $f\left(x_{1}\right)$ and $f\left(x_{2}\right)$ have to be evaluated.

\subsection{Optimization of a Single Threshold}

Let us consider the case of 2 segments. In this case, we have only a single threshold to determine.

Figure 5 presents the evolution of GS search over the intermediate points $x_{1_{(q)}}$ and $x_{2_{(q)}}$ in the two first iterations and the final iteration. The blue curve presents the NMSE of postdistortion for every position of the threshold between 0 and 1 . The red curve presents the AM-AM characteristic of the PA.

Initially, the search interval $[a b]=\left[\begin{array}{ll}0 & 1\end{array}\right]$. At the first iteration, the search interval $[a b]$ is updated with the narrower interval $\left[\begin{array}{ll}a_{1} & 1\end{array}\right]$. After 34 iterations, $\left[\begin{array}{ll}a_{34} & b_{34}\end{array}\right]$ fulfills the condition $\left|a_{34}-b_{34}\right|<\epsilon$ that allows the GS search to stop and return $x_{\mathrm{opt}}=\frac{a_{34}+b_{34}}{2}$ as the optimized threshold.

Thus, the NMSE from uniform segmentation to optimal segmentation is improved by nearly $5 \mathrm{~dB}$, as it can be seen from Fig. 5 .

\subsection{General Case with K Segments}

In the general case, for $K$ segments, we have $K-1$ thresholds to optimize: $\beta=\beta_{1}, \beta_{2}, \ldots, \beta_{K-1}$. We propose to optimize the thresholds successively one by one, starting with an initial uniform segmentation. The optimization interval $I_{i}$ of a selected $\beta_{i}$ is $\left[\beta_{i-1} \beta_{i+1}\right]$. In a normalized interval, $\beta_{0}=0$ and $\beta_{K}=1$ by definition.

Each $\beta_{i}$ contribute to the overall NMSE, furthermore as $\beta_{i}$ is the bound of the optimization interval for $\beta_{i-1}$ and $\beta_{i+1}$ so the optimization of $\beta_{i}$ will in turn impact the optimal 
position of $\beta_{i-1}$ and $\beta_{i+1}$ that should be re-evaluated. That is why we propose a heuristic to handle this situation: after having optimized the last threshold $\beta_{K-1}$, the algorithm is run in reverse order back to $\beta_{1}$, then again up to $\beta_{K-1}$, and so on until the segmentation has converged.

Given the DVR model in (1) defined by $K, M$ and the thresholds $\beta_{1}, \cdots, \beta_{K-1}$. The function $\operatorname{NMSE}\left(\beta_{i}\right)$ corresponds to the different value of NMSE, obtained when varying $\beta_{i}$ from $\beta_{i-1}$ to $\beta_{i+1}$. The minimum of this function is obtained with $\operatorname{argmin} \operatorname{NMSE}\left(\beta_{i}\right)$, where:

$$
\beta_{i} \in\left[\beta_{i-1}, \beta_{i+1}\right]
$$

The algorithm for the proposed approach is described in Algorithm (2) in which the steps $\beta_{K-1}(q)=\beta_{K-1}(q-1)$ and $\beta_{1}(q)=\beta_{1}(q-1)$ are introduced for notation consistency.

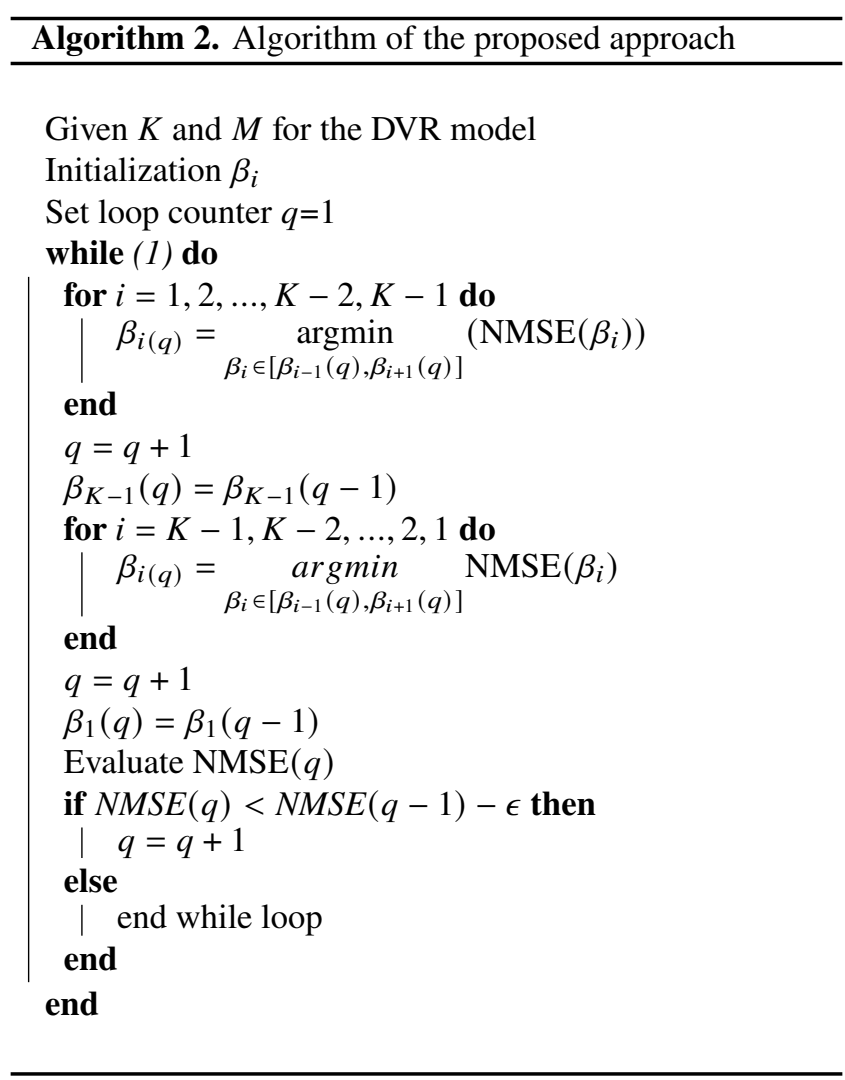

\subsection{Complexity of GS Search}

The complexity of the GS search is an important aspect to be considered in the thresholds optimization process.

In this paper, the search complexity is assessed by the number of NMSE computations, which require to estimate the coefficients. The number of GS iterations is defined by the number of times the search interval is updated, except at the first iteration, one GS iteration corresponds to one NMSE evaluation.

\section{Performance of the Proposed Ap- proach}

\subsection{Parameters of DVR Model}

In the following, we set the number of segments $K$ to 4 and the memory depth $M$ to 2 . We use the DVR model presented in (1). The number of coefficient is 51 .

\subsection{Comparison of Linearization Performances}

In this section, the proposed approach results are discussed and compared to those of a uniform segmentation and the optimal segmentation obtained from brute-force search and GA algorithm. The results are summarized in Table 1 and discussed in terms of NMSE and ACPR, where L1 and U1 refer to the lower and upper adjacent channels and L2 and U2 lower and upper alternate channels. The algorithm complexity is also discussed and evaluated by the number of computations of the objective function.

The first column corresponds to the uniform segmentation. The next column represents brute-force search, which has been performed by dividing the input range into 100 steps. The third column represents GA's results, which have been performed with a population size of up to 50 and a maximum number of generations up to 25 . The last column presents the results of the proposed approach.

In the scenario at hand, the proposed approach has converged towards quasi the same optimal segmentation returned by brute-force search and GA. According to Tab. 1, only 248 objective function evaluations are performed to optimize the thresholds, while GA requires 2554 evaluations and bruteforce requires 161700 evaluations to find the same optimal results.

Compared to uniform segmentation, the NMSE of the proposed approach has been significantly improved by nearly $6.5 \mathrm{~dB}$, as it can also be seen from Fig. 4 for $K=4$.

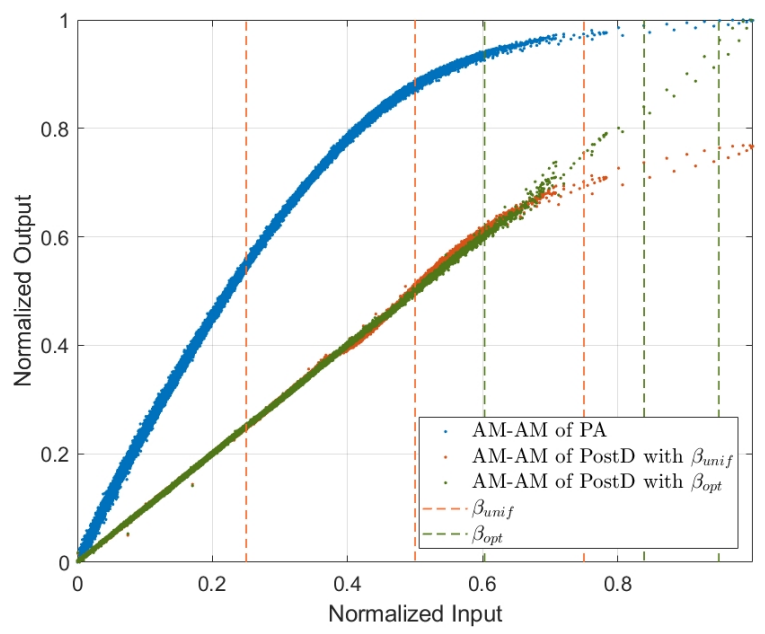

Fig. 6. AM/AM plots of PA with uniform and optimized segmentation. 


\begin{tabular}{|c|c|c|c|c|c|}
\cline { 2 - 6 } \multicolumn{1}{c|}{} & $\begin{array}{c}\text { Uniform } \\
\text { segmentation }\end{array}$ & Brute-force & GA & $\begin{array}{c}\text { Proposed approach } \\
\text { with uniform segmentation }\end{array}$ \\
\hline \multicolumn{2}{|c|}{ Segmentation } & {$[0.250 .50 .75]$} & {$[0.600 .830 .94]$} & {$[0.600 .830 .94]$} & {$[0.600 .840 .95]$} \\
\hline \multicolumn{2}{|c|}{ NMSE (dB) } & -34 & -40.43 & -40.43 & -40.43 \\
\hline \multirow{4}{*}{ ACPR(dB) } & L2 & -52.53 & -56.85 & -56.86 & -56.85 \\
\cline { 2 - 6 } & L1 & -41.39 & -43.51 & -43.49 & -43.51 \\
\cline { 2 - 6 } & U1 & -42.15 & -44.07 & -44.08 & -44.07 \\
\cline { 2 - 6 } & U2 & -53.34 & -58.45 & -58.55 & -58.44 \\
\hline \multicolumn{2}{|c|}{ Complexity } & 1 & 161700 & 2554 & 248 \\
\hline
\end{tabular}

Tab. 1. Comparison of linearization performances, optimal solutions and complexity

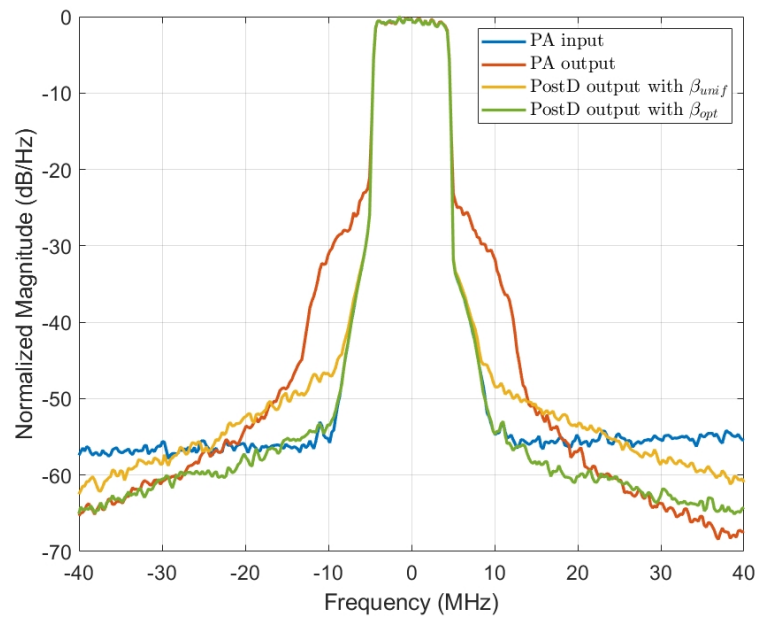

Fig. 7. Power spectrum of $10 \mathrm{MHz}$ LTE signal where is the input and output of PA and output of Post-D with DVR model with uniform and optimized segmentation.

The linearization performances of the optimal segmentation with $\beta_{\mathrm{opt}}=\left[\begin{array}{lll}0.60 & 0.84 & 0.95\end{array}\right]$ are presented in Fig. 6 and Fig. 7 where they are compared to that of the uniform segmentation with $\beta_{\text {unif }}=[0.250 .50 .75]$. Figure 6 presents the AM/AM characteristics of the PA and that of the predistorters with uniform and optimized segmentation. It can be seen that the AM-AM curve is improved with optimal segmentation compared to uniform segmentation. The power spectrum of the output of the postdistorter for DVR model with uniform and optimized segmentation are shown in Fig. 7. The ACPR with $\beta_{\text {opt }}$ has been significantly improved than the DVR model with $\beta_{\text {unif. }}$.

\subsection{Analysis of the Proposed Approach}

This section highlights the behavior of the proposed approach throughout its convergence and how the optimization problem is decomposed into a set of unimodal sub-problems. Furthermore, a stochastic process is proposed to select the initial segmentation to accelerate the convergence of the proposed approach.

\subsubsection{Behavior of the Proposed Approach}

The initial segmentation is uniform, i.e. $\beta=$ $\left[\begin{array}{lll}\beta_{1(0)} & \beta_{2(0)} & \beta_{3(0)}\end{array}\right]=\left[\begin{array}{lll}0.25 & 0.5 & 0.75\end{array}\right]$. The optimization interval vector is $I_{(0)}=\left[I_{1(0)} ; I_{2(0)} ; I_{3(0)}\right]=$ [[0 0.5$] ;\left[\begin{array}{ll}0.25 & 0.75\end{array}\right] ;\left[\begin{array}{ll}0.5 & 1\end{array}\right]$. Figure 8 presents the behavior of $\operatorname{NMSE}\left(\beta_{i}\right)$ on each interval. It can be seen from Fig. 9 that $\operatorname{NMSE}\left(\beta_{1}\right)$ is not unimodal on $I_{1(0)}$.

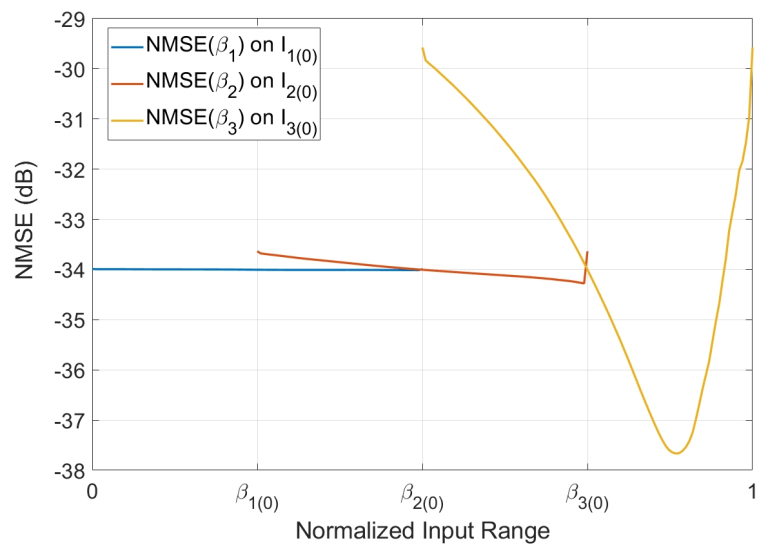

Fig. 8. $\operatorname{NMSE}\left(\beta_{i}\right)$ on $I_{i}(0)$.

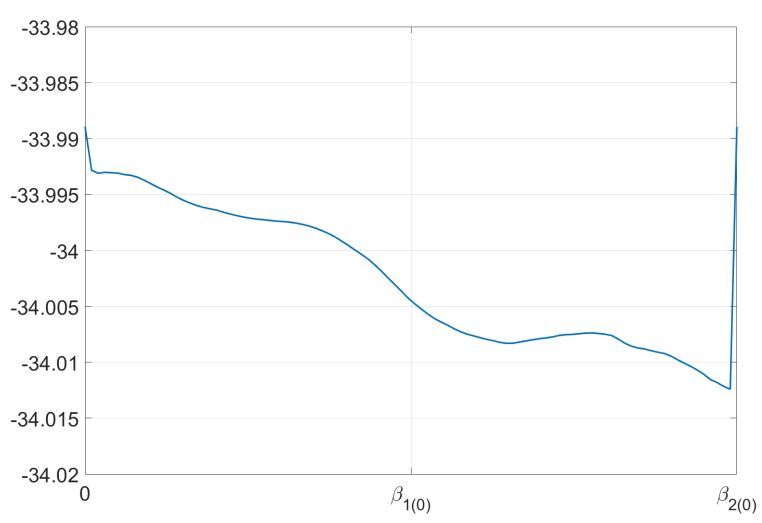

Fig. 9. Zoom of $\operatorname{NMSE}\left(\beta_{1}\right)$ on $I_{1(0)}$.

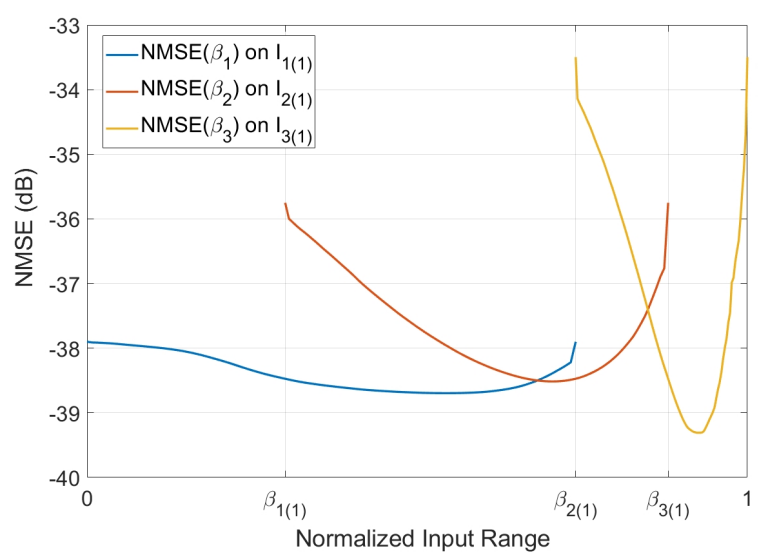

Fig. 10. $\operatorname{NMSE}(\beta)$ on $\boldsymbol{I}_{(1)}$. 


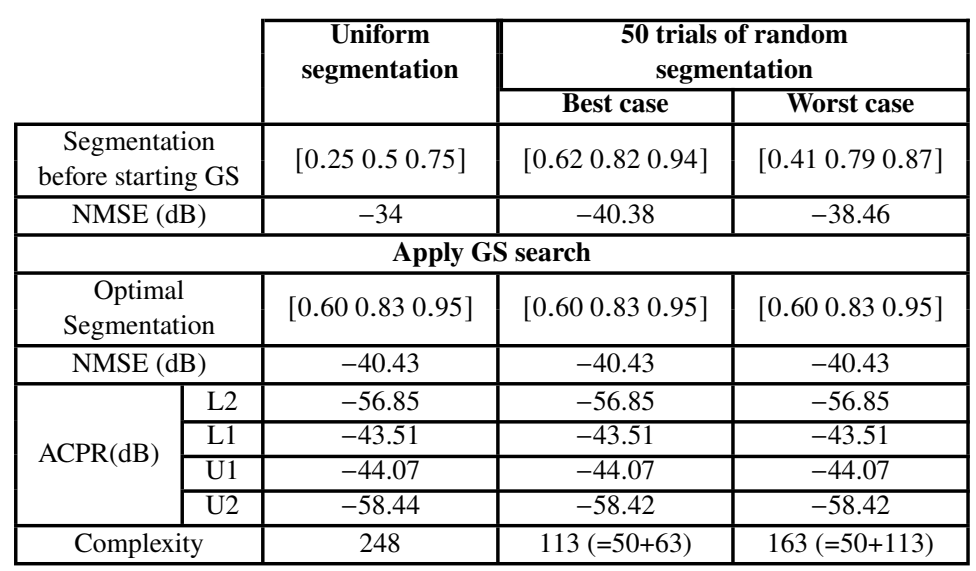

Tab. 2. Comparison of linearization performances, optimal solutions and complexity with random selection.

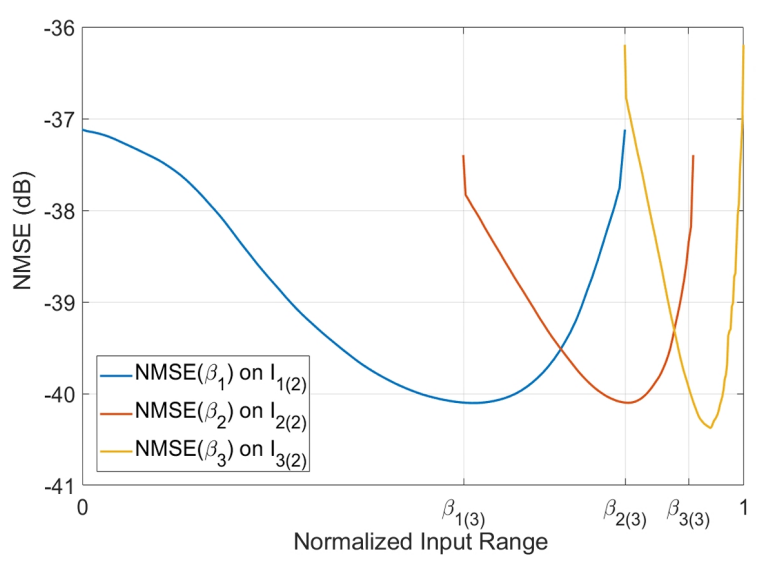

Fig. 11. $\operatorname{NMSE}(\beta)$ on $I_{(2)}$.

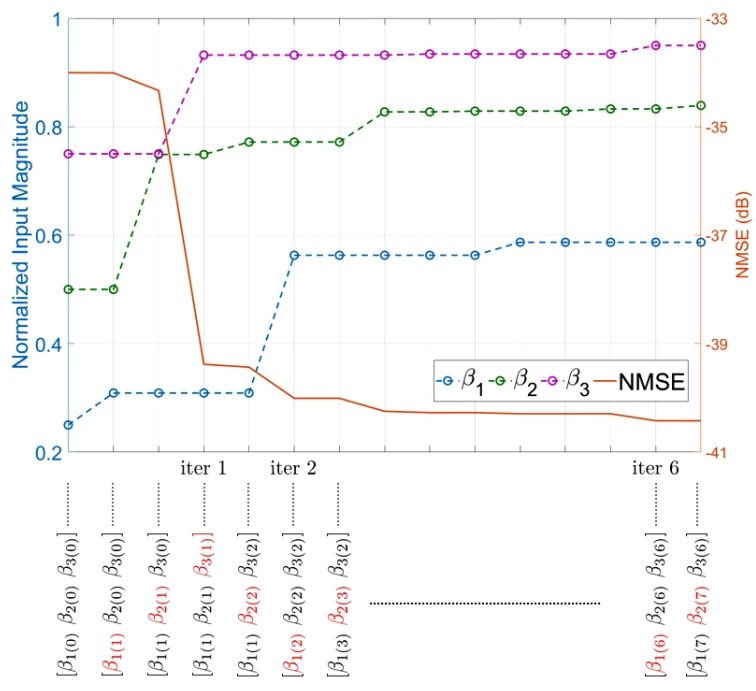

Fig. 12. Evolution of thresholds and NMSE Versus iterations.

After the optimization of $\left[\begin{array}{lll}\beta_{1(0)} & \beta_{2(0)} & \beta_{3(0)}\end{array}\right]$ to $\left[\begin{array}{lll}\beta_{1(1)} & \beta_{2(1)} & \beta_{3(1)}\end{array}\right]=\left[\begin{array}{lll}0.3 & 0.74 & 0.88\end{array}\right]$, The behavior of NMSE on the updated optimization interval vector $I_{(1)}=$ $\left[I_{1(1)} ; I_{2(1)} ; I_{3(1)}\right]=\left[\left[\begin{array}{ll}0 & 0.74\end{array}\right] ;\left[\begin{array}{ll}0.3 & 0.88\end{array}\right] ;\left[\begin{array}{lll}0.74 & 1\end{array}\right]\right.$ is presented in Fig. 10 which shows that the behavior of NMSE on $I_{1(1)}$ becomes unimodal. Hence this example highlights the following interesting behavior, even if each sub-problems are not unimodal at the beginning, they become unimodal as each sub-problem converges towards its optimum. After the second iteration, we get $\left[\beta_{1(2)} \beta_{2(2)} \beta_{3(2)}\right]=\left[\begin{array}{lll}0.57 & 0.78 & 0.91\end{array}\right]$, for which the functions $\operatorname{NMSE}\left(\beta_{i}\right)$ are shown in Fig. 11.

Figure 12 presents the evolution of thresholds $\beta_{1}, \beta_{2}$, $\beta_{3}$ and NMSE where the optimization of thresholds has been performed in direct order: $\left[\beta_{1} ; \beta_{2} ; \beta_{3} ; \beta_{2} ; \beta_{1} ; \beta_{2} ; \ldots\right]$, until the algorithm has converged.

\subsubsection{Random Selection}

In Sec. 5.3.1, It has been shown that starting from a uniform segmentation, some of the sub-problems to solve may not be unimodal. Even though if it does not prevent the convergence of the algorithm, the choice of the initial segmentation may be essential to start with a segmentation that can give a unimodal criterion on each interval from the first iteration, which could speed up the convergence of the algorithm and decrease the execution time.

For that, a stochastic selection process is proposed: $N$ trials of random segmentation are performed, and the best one in terms of NMSE is retained as the initial segmentation for the proposed algorithm.

To validate the effectiveness of the stochastic process, 100 experiments of threshold optimization with random selection have been performed for $N=50$.

Table 2 highlights that GS search requires 248 GS iterations starting from a uniform segmentation while it requires only 113 or 163 GS iterations for the best and worst case of the 100 experiments considered.

As the number of GS iterations becomes a random variable using stochastic initialization, it is necessary to look for the probability distribution function that best fits the experimental data to determine an upper bound on the expected number of GS iterations.

It turns out that a Burr distribution [13] with a mean of 80 and a variance of 116 fits well the experimental data. With these parameters, the upper bound on the number of GS iterations is 120 with a probability of 0.99 and 154 with a probability of 0.999 . 


\begin{tabular}{|c|c|c|c|c|}
\hline \multirow{2}{*}{} & \multirow{2}{*}{ Uniform Seg. } & \multicolumn{3}{|c|}{ Stochastic initialization with 50 trials } \\
\cline { 3 - 5 } & & Mean & $p=0.99$ & $p=0.999$ \\
\hline Initialization & - & \multicolumn{3}{|c|}{50} \\
\hline GS & 248 & 80 & 120 & 154 \\
\hline Total & 248 & 130 & 170 & 204 \\
\hline
\end{tabular}

Tab. 3. Complexity comparison. Here $p$ is the probability that the required number of GS iterations is below or equal to the given value.

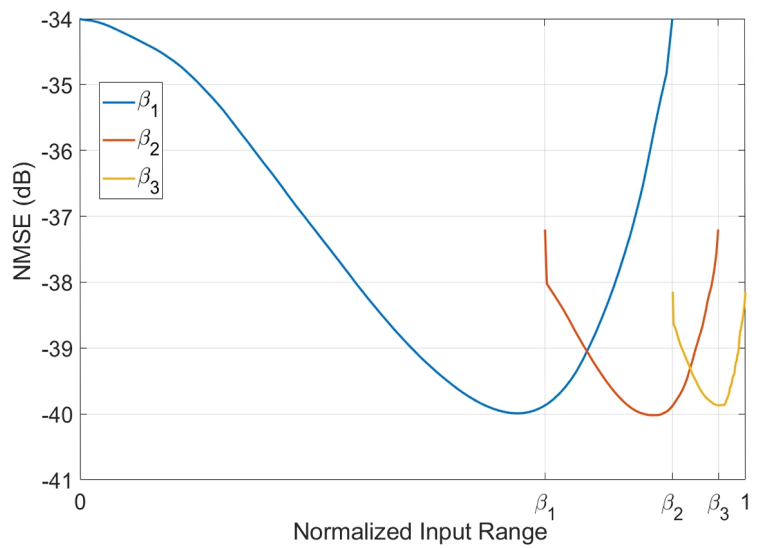

Fig. 13. Variation of NMSE on intervals $I_{1}, I_{2}$ and $I_{3}$ with random selection

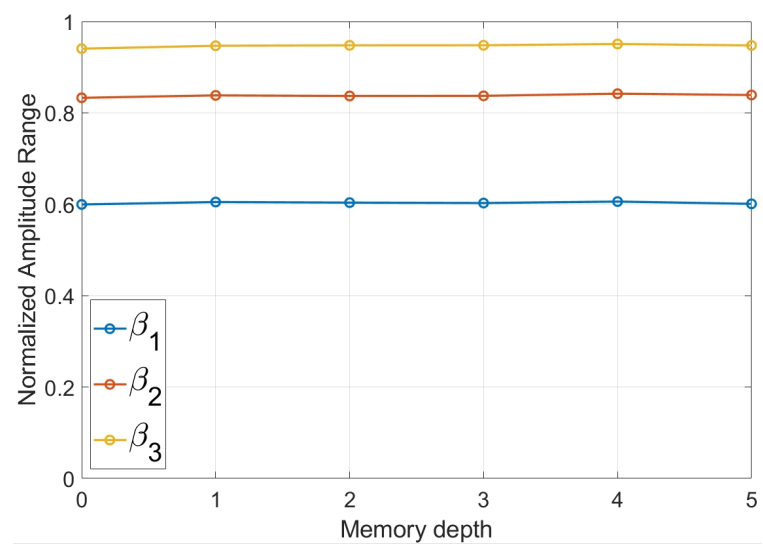

Fig. 14. Optimal segmentation vs Memory depth $M$.

Thus, using a stochastic initialization with 50 trials, the average number of GS iterations will be 80 and below 120 with a probability of 0.99 or 154 with a probability of 0.999 . Those results are summarized in Tab. 3.

Furthermore, after the random selection process, the behavior of each $\operatorname{NMSE}\left(\beta_{i}\right)$ on their interval $I_{i(0)}$ are likely to be unimodal before starting the GS algorithm, as it can be seen in Fig. 13. Experiments have shown that some tens of random selection trials are likely to provide a starting point that improves the NMSE significantly to the uniform segmentation. It is worth noting that for every 100 experiments of the stochastic initialization, the proposed optimization approach has converged towards the same segmentation. This illustrates its convergence behavior.

\subsection{Sensitivity of Thresholds to Memory Depth}

Figure 14 compares each threshold's optimal position for different memory depth $M$ with $K=4$, for the same scenario. This highlights an interesting behavior. The optimal segmentation is not very sensitive to memory depth $M$. So that, the optimization can be performed for the DVR model without memory in order to reduce the algorithm complexity, which makes it an important aspect to design an optimal DVR model.

\section{Conclusion}

This paper showed that the optimal segmentation could significantly improve the linearization performances compared to the uniform segmentation for the DVR model. We have proposed a new approach for optimizing the thresholds based on the decomposition of the global optimization problem into a set of sub-problems where the objective function is unimodal. The golden section algorithm is used to solve each unimodal sub-problem. The proposed approach has been compared with the genetic algorithm. Both algorithms converge to quasi the same values of thresholds. Nevertheless, our approach presents a much lower complexity than the GA algorithm making it an appropriate candidate to design the DVR model.

\section{Acknowledgments}

Research reported in this publication is part of the APOGEES project supported by BPI France, region Occitanie and region Nouvelle Aquitaine. APOGEES project has been labeled by from pole Aerospace Valley, pole Images et Reseaux and pole Elopsys in the framework of the French FUI22 research program.

\section{References}

[1] KATZ, A., WOOD, J., CHOKOLA, D. The evolution of pa linearization: from classic feedforward and feedback through analog and digital predistortion, IEEE Microwave Magazine, 2016, vol. 17, no. 2, p. 32-40. DOI: 10.1109/MMM.2015.2498079

[2] JABBOUR, C., DESGREYS, P., DALLET, D. Digitally Enhanced Mixed Signal Systems. Stevenage (UK): Institution of Engineering and Technology, 2019. ISBN: 9781785616099

[3] DING, L., ZHOU, G. T., MORGAN, D. R., et al. A robust digital baseband predistorter constructed using memory polynomials. IEEE Transactions on Communications, 2004, vol. 52, no. 1, p. 159-165. DOI: 10.1109/TCOMM.2003.822188

[4] MORGAN, D.R., ZHENGXIANG, M., JAEHYEONG K., et al. A generalized memory polynomial model for digital predistortion of 
rf power amplifiers. IEEE Transactions on Signal Processing, 2006, vol. 54, no. 10, p. 3852-3860. DOI: 10.1109/TSP.2006.879264

[5] ZHU, A., PEDRO, J. C., BRAZIL, T. J. Dynamic deviation reductionbased Volterra behavioral modeling of $\mathrm{rf}$ power amplifiers. IEEE Transactions on Microwave Theory and Techniques, 2006, vol. 54, no. 12, p. 4323-4332. DOI: 10.1109/TMTT.2006.883243

[6] KANTANA, C., VENARD, O., BAUDOIN, G. Comparison of GMP and DVR models. 2018 International Workshop on Integrated Nonlinear Microwave and Millimetre-wave Circuits (INMMIC), 2018, p. 1-3. DOI: 10.1109/INMMIC.2018.8430008

[7] ZHU, A. Decomposed vector rotation-based behavioral modeling for digital predistortion of rf power amplifiers. IEEE Transactions on Microwave Theory and Techniques, 2015, vol. 63, no. 2, p. 737-744. DOI: 10.1109/TMTT.2014.2387853

[8] CAVERS, J. K. Optimum table spacing in predistorting amplifier linearizers. IEEE Transactions on Vehicular Technology, 1999, vol. 48, no. 5, p. 1699-1705. DOI: 10.1109/25.790551

[9] MAGESACHER, T., SINGERL, P., MATALN, M. Optimal segmentation for piecewise rf power amplifier models. IEEE Microwave and Wireless Components Letters, 2016, vol. 26, no. 11, p. 909-911. DOI: 10.1109/LMWC.2016.2614974

[10] MATEO, C., CARRO, P., GARCIA-DUCAR, P., et. al. Radioover-fiber linearization with optimized genetic algorithm CPWL model. Optics Express, 2017, vol. 25, p. 3694-3708. DOI: 10.1364/OE.25.003694

[11] CHUA, L. O. Section-wise piecewise-linear functions: canonical representation, properties, and applications. Proceedings of the IEEE, 1977, vol. 65, no. 6, p. 915-929. DOI: 10.1109/PROC.1977.10589

[12] KIEFER, J. Sequential minimax search for a maximum. Proceedings of the American Mathematical Society, 1953, vol. 4, no. 3, p. 502-506. DOI:10.2307/2032161

[13] BURR, I. W. Cumulative frequency functions. The Annals of Mathematical Statistics, 1942, p. 215-232. DOI:10.1214/aoms/1177731607

\section{About the Authors ...}

Chouaib KANTANA received his M.S. degree from Cadi Ayyad University, Marrakesh, Morocco, in 2016. He is currently a Ph.D. student with ESYCOM, University Gustave Eiffel, Marne-la-Vallée, France. His current research interests include wireless communications, digital predistortion, optimization problems, deep learning for for wireless communication systems.

Geneviéve BAUDOIN received graduate engineering degree from Ecole Nationale Superieure des Telecommunications, Paris, France in 1977 and the habilitation for Ph.D. direction from the University of Marne La Vallee, Marne La Vallee, France, in 2000. She was a lecturer at the University of Paris-Ouest before she joined the Philips Research laboratory in France as a research-engineer and worked on ultrasound signal processing for medical applications. She is currently Professor at ESIEE Paris, France. Her research and teaching activities include digital signal processing, wireless communications, wireless transmitter linearization, transceiver architectures and algorithms for wireless communications and localization systems.

Olivier VENARD received the M.S. degree in Electronics, Sensors, and Signal Processing from Universite Paris-Sud, Orsay, France, in 1996. He was with the Center National d Etude des Telecommunication (Paris), where he was involved in network technologies and embedded electronics. Since 1997, he has been an Assistant Professor with ESIEE Paris, Noisy-le-Grand, France. He is currently a member of the ESYCOM Laboratory, Universite Paris-Est, Champs-surMarne, France. His current research and teaching interests include signal processing algorithms for sensors and digital communications, nonlinear signal processing for linearization, adaptive signal processing, wireless communications, networks protocols, embedded electronics, and Internet of Things. 